\title{
Experimental Study on the Bending Properties of Functionally Graded Shape Memory Alloy Laminate
}

\author{
Bingfei Liu ${ }^{1, *}$, Anmin $\mathrm{Zhao}^{2}$ and Chunzhi $\mathrm{Du}^{1}$ \\ ${ }^{1}$ Airport College, Civil Aviation University of China, Tianjin, 300300, China \\ ${ }^{2}$ Faculty of Aerospace Engineering, Civil Aviation University of China, Tianjin, 300300, China \\ ${ }^{*}$ Corresponding author
}

\begin{abstract}
An experimental work is obtained for the test force displacement curve of functionally graded Shape Memory Alloy (SMA) laminate subjected to three point bending test. The laminate is uniform and composed by five layers including three SMA layers and two ceramic layers. The relationship between the test force and the displacement for the functionally graded shape memory alloy laminate subjected to three point bending test is finally given.
\end{abstract}

Keywords-shape memory alloy; functionally graded material; experimental work

\section{INTRODUCTION}

The Shape Memory Alloys (SMAs) show a variety of remarkable engineering properties, most notabley the shape memory effect and pseudoelasticity $[1,2]$. They are used in a wide range of applications including actuation mechanisms $[3,4]$. Due to the unique behaviors such as special shape memory effect, superelasticity and good biocompatibility of SMAs, this list of applications continues to expand.

Mechanics of Functionally Graded Materials (FGMs) have been extensively studied by researchers in the past two decades [5]. Due to the ability of FGMs to solve such complicated problems, these materials have been explored for engineering applications in thermal, structural, optical and electronic materials [6-7].

For more excellent mechanical properties, the Functionally Graded Shape Memory Alloy (FG-SMA) composites, as a special case of the FGMs using SMA as the metal phase, are being developed incorporating a wide variety of matrices [8]. By developing such a composite, the material can meet the requirement of high temperature conditions and possess higher mechanical properties [9]. The artificial compressive residual stress and the mechanical properties of composites will be improved significantly [10]. Besides, these materials have great promise in the biomedical area where it can be used for hard tissue replacement [11]. Furthermore, these composites have great potential to be used in new sensor technology, information technology and the emerging field of smart materials systems [12-13].

In this paper, the mechanic behaviors including the theoretical solution and the experimental result are given. Firstly, the experimental result for the test force displacement curve of functionally graded shape memory alloy laminate subjected to three point bending test is given. The bending modulus of the laminate is given and combing with the constitutive model of the SMA, the theoretical result of the relationship between the test force and the displacement is then obtained. At last, the two results are compared with each other..

\section{EXPERIMENTAL WORK}

Consider a five-layered system made up of a compositionally grade layer in between a homogeneous ceramic material and an SMA material. As shown in Fig. 1, the up layer is considered as the SMA and the second layer is considered as the homogeneous ceramic, the mid layer and the lower layer are SMA and the forth layer is the homogeneous ceramic. The system is stress free at the initial case and the stress free is kept on all boundary surfaces.

Let the total length, width and height of the multilayer system be given by $l, w$ and $h$, respectively, see Fig. 1(a). The geometry of the model allows it to be idealized as an equal biaxial stress plate, and the variables of interest depend only on the out of plane coordinate $y$. The in-plane geometry of the layered structure is shown in Fig. 1(b). The interfaces between the different layers are assumed to be perfectly bonded at all times.

The materials are purchased from Beijing Jiyikemao limited company. The laminate is composed by five uniform layers including 3 SMA layers and 2 ceramic layers with equal thickness. The initial thick of each layer are $3 \mathrm{~mm}$, and the final thick of the whole laminate is $1 \mathrm{~mm}$ after the high-temperature pressing. The functionally graded SMA laminate sample can be shown in Fig. 2. The dimensions of sample are $100 \times 2 \times 150 \mathrm{~mm} 3$.

The bending properties of the functionally graded SMAs were determined by the load-distortion curve obtained from the three-point bending test. The sketch of the three point bending test can be seen in Fig. 3. And the experiment device is the MTS universal tester. Then start the experiment with the loading rate is $0.0005 \mathrm{in} / \mathrm{sec}$, and the maximum loading is $95 \mathrm{KN}$, then unloading. The relationship between the test force and the displacement of the functionally graded SMA laminate is then obtained in Fig. 4. As seen in Fig. 4, the elastic stage of the functionally graded SMA laminate shows not a straight line, and the strengthen stage is also show an indiscipline curve. 

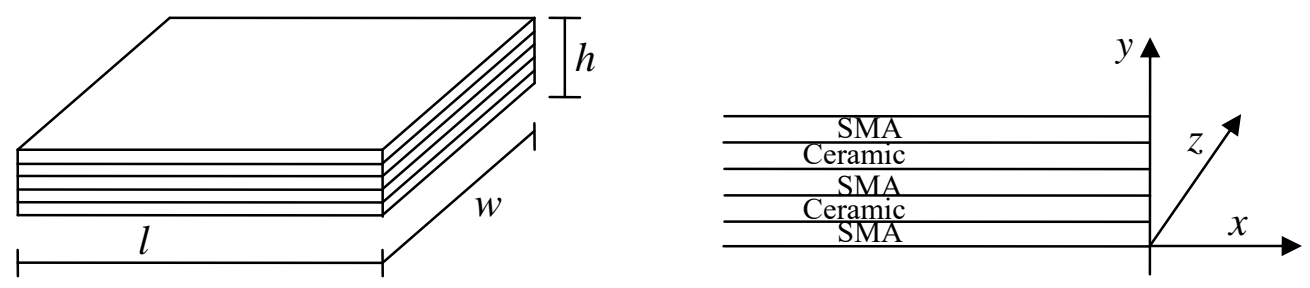

FIGURE I. (A) FIVE-LAYERED STRUCTURAL MODEL AND (B) COORDINATE AXES AND DIMENSIONS OF THE FIVE-LAYERED LAMINATE SYSTEM.
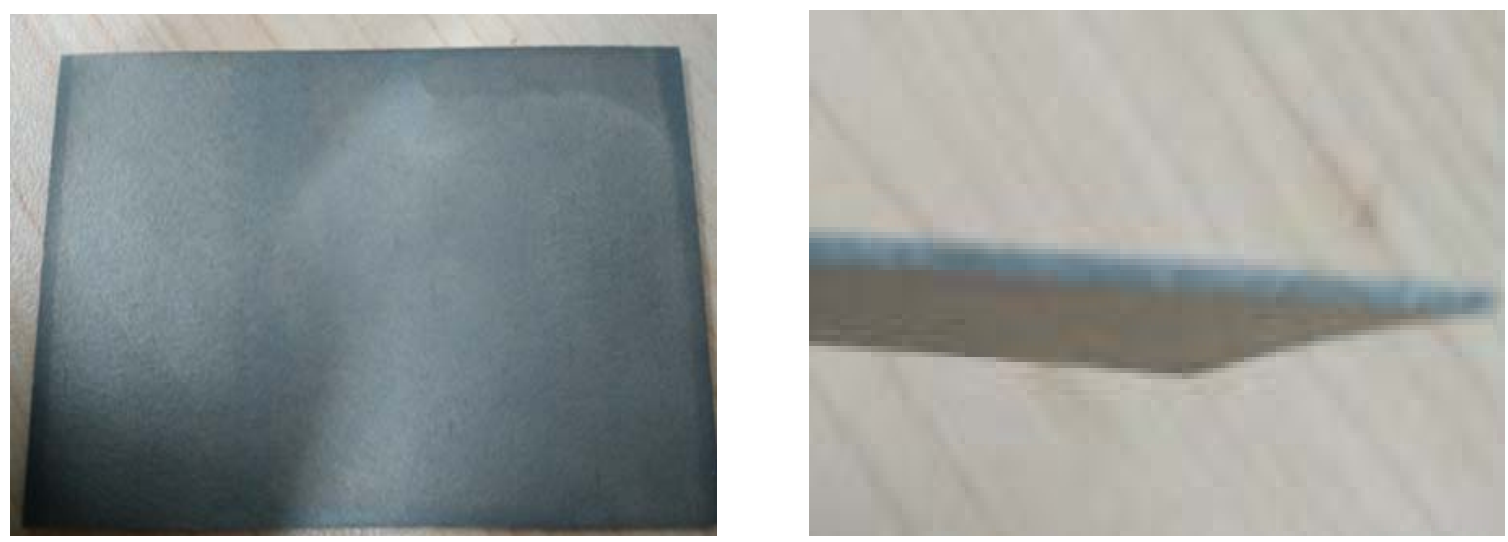

FIGURE II. (A) THE FUNCTIONALLY GRADED SMA LAMINATE SAMPLE AND (B) THE SECTION VIEW OF THE FUNCTIONALLY GRADED SMA LAMINATE SAMPLE

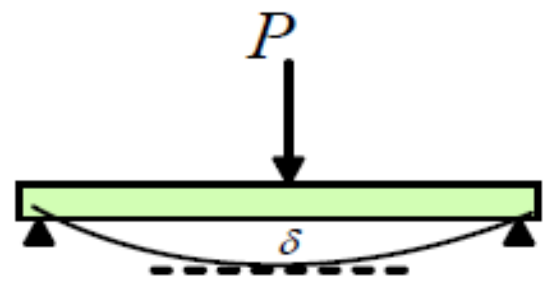

FIGURE III. THE SKETCH OF THE THREE POINT BENDING TEST

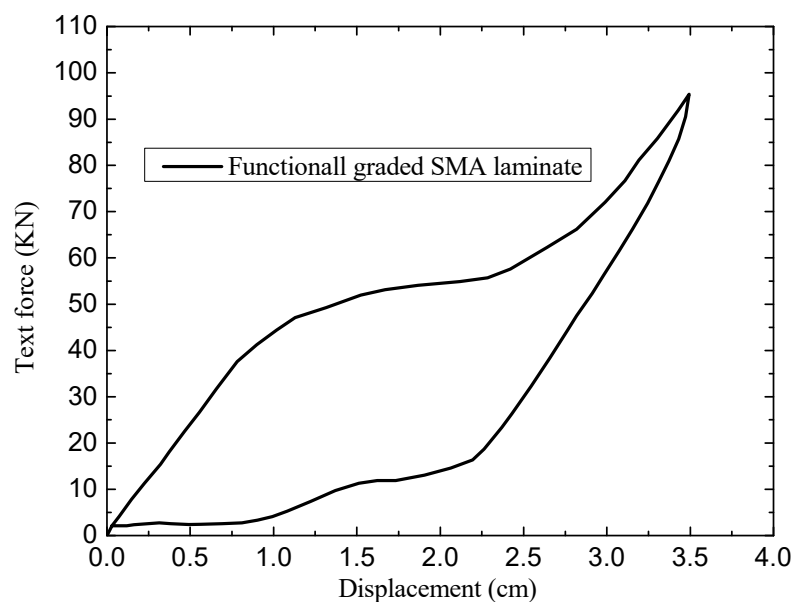

FIGURE IV. THE RELATIONSHIP BETWEEN THE TEST FORCE AND THE DISPLACEMENT OF THE FUNCTIONALLY GRADED SMA LAMINA 


\section{CONCLUSIONS}

A five-layered system made up of a compositionally grade layer in between a homogeneous ceramic material and an SMA material is first designed in this work. The entity functionally graded SMA laminate which composed by five uniform layers including 3 SMA layers and 2 ceramic layers with equal thickness is then produced by high-temperature pressing. Using the MTS universal tester, the relationship between the test force and the displacement of the functionally graded SMA laminate under three-point bending test is finally obtained. The results show that the elastic stage of the functionally graded SMA laminate is not a straight line, and the strengthen stage is also indiscipline.

\section{ACKNOWLEDGMENT (HEADING 5)}

The authors acknowledge the financial support of National Natural Science Foundation of China (No. 11502284; 51505483) and the financial support of Tianjin Natural Science Foundation (No. 15JCQNJC42600) and it is also supported by "the Fundamental Research Funds for the Central Universities of China (2016: Analysis of fatigue, damage and failure of the variable geometry jet engine chevron).

\section{REFERENCES}

[1] Yinong Liu, S.P. Galvin., Criteria for pseudoelasticity in nearequiatomic NiTi shape memory alloys. Acta Materialia, 1997, 45: 44314439.

[2] Humbeeck J. V., Shape Memory Alloys: a material and a technology. Advanced Engineering Materials 2001, 3: 837-850.

[3] Humbeeck J. V., Non-medical application of shape memory alloys . Material. Science. Engineering. A,1999,273-275: 134-148.

[4] Bellouard Y., Shape memory alloys for microsystems: A review from a material research perspective. Materials. Science. Engineering. A 2008 481-482:582-589.

[5] B.A.S Shariat., R. Javaheri. and M. R. Eslami., Buckling of imperfect functionally graded plates under in-plane compressive loading, ThinWalled Structures, 2005, 43: 1020-1036.

[6] Shao, Z.S., and Wang, T.J., Three-dimensional solutions for the stress fields in functionally graded cylindrical panel with finite length and subjected to thermal mechanical loads. International Journal of Solids and Structures, 2006, 43:3856-3874.

[7] Wang, J.P., Chen, G. and Zhai, P.C., Creep property of functionally graded materials. Materials Science Forum, 2005, 492-493: 441-446.

[8] Liu, B.F., Dui, G.S. and Yang, S,Y., On the transformation behavior of functionally graded SMA composites subjected to thermal loading. European Journal of Mechanics A-solids, 2013, 40: 39-147.

[9] Shabana, Y.M. and Noda, N., Thermo-elasto-plastic stresses in functionally graded materials subjected to thermal loading taking residual stresses of the fabrication process into consideration. Composites: Part B, 2001, 32: 111-121.

[10] Miyazaki, E and Watanabe, Y., Development of shape memory alloy fiber reinforced smart FGMs. Materials Science Forum, 2003, 423-425: 107-112.

[11] Zhang, Y.P., Zhang, X.P. and Zhong, Z.Y., Fabrication, transformation and superelasticity behavior of NiTi memory alloy with large pore-size and gradient porosity. Aata Metallurgica Sinica, 2007, 43(11): 12211227.

[12] Mahmud, A.S., Liu, Y.N. and Nam, T.H., Gradient anneal of functionally graded NiTi. Smart Material \& Structures, 2008, 17: 1-5.

[13] B.Skoczeń., Functionally graded structural members obtained via the low temperature strain induced phase transformation. International Journal of Solids and Structures, 2007, 44: 5182-5207 